ARTÍCULO DE ECONOMÍA AMBIENTAL 



\title{
EL MARCO CATEGORIAL DE LA CRISIS AMBIENTAL EN UN CONTEXTO GLOBALIZADO
}

\author{
THE CATEGORIAL FRAMEWORK OF THE ENVIRONMENTAL CRISIS IN A GLOBAL \\ CONTEXT \\ Ofelia Agoglia ${ }^{1}$ \\ RESUMEN
}

El trabajo desarrollado se estructura en torno a la construcción de un marco categorial a partir del cual se propone una síntesis de la posición adoptada por el pensamiento ambiental crítico, en cuanto al análisis de las características específicas que adopta la crisis ambiental en el marco del proceso globalizador.

A partir de este análisis se propone inferir la relación causal entre las características de la modernidad avanzada y la profundización de la crisis ambiental, teniendo en cuenta las contradicciones que se vislumbran entre sus manifestaciones crecientes y las dificultades que conlleva la implementación de estrategias alternativas de solución, considerando la capacidad del sistema de integrar sus elementos distorsivos a través de dispositivos o mecanismos de reproducción, desde los cuales, las relaciones de poder subyacentes al proceso de ruptura ambiental se mantienen inalterables.

Palabras clave: análisis categorial, crisis, modernidad, pensamiento ambiental crítico, ruptura.

\begin{abstract}
The present work is structured around the construction of a set of categories from which to propose a synthesis of the stance taken by critical environmental thought regarding the analysis of the features of the environmental crisis that are specific to the globalization process.

This analysis intends to infer the relationship between the characteristics of advanced modernity and the deepening of the environmental crisis. It takes into account the contradictions that can be envisaged between its increasingly apparent symptoms and the difficulties involved in implementing alternative strategies for possible solutions, considering the capacity of the system to integrate its own distorting elements through mechanisms of reproduction, as a consequence of which the underlying power relations of the process of environmental disruption remain unchanged.
\end{abstract}

Key words: Categorial Analysis, crisis, modernity, critical environmental thought, break.

Recibido: 6 de noviembre de 2012 Aceptado: 20 de diciembre de 2012

1- Universidad Nacional de Cuyo, Mendoza Argentina.

Doctora en Educación Ambiental - Correo electrónico: oagoglia@uncu.edu.ar - Tel: 00542614987071. Articulación de reflexión.. 


\section{INTRODUCCIÓN}

A partir del presente trabajo se propone abordar la relación causal entre las dimensiones críticas de la modernidad avanzada y la profundización de la crisis ambiental, a través de la conformación de un marco categorial cuya construcción se deriva de las contribuciones teóricas vertidas por referentes del pensamiento ambiental crítico, que focalizan su análisis en las características específicas que adopta la crisis ambiental en el marco del proceso globalizador, considerando la capacidad del sistema de subsumir las propuestas críticas a través de dispositivos de reproducción, desde los cuales, las relaciones de poder subyacentes al proceso de ruptura ambiental se mantienen inalterables.

Los conceptos vertidos constituyen un recorte de los resultados alcanzados a partir de la investigación desarrollada en mi trabajo de tesis doctoral (Agoglia, O. (2011). Desde la perspectiva teórica considerada, la crisis ambiental se concibe como parte de un proceso sociohistórico amplio que sólo puede ser analizado en el marco de un contexto de crisis estructural, que remite a la ruptura del proyecto moderno como consecuencia de la racionalización de la sociedad occidental (Agoglia, 2011).

El virtud del objeto de estudio, se recurre a una perspectiva teórico-metodológica que responde a un enfoque sociocrítico ${ }^{2}, a$ partir de la cual se pretende ir más allá de lo aparente, e indagar acerca de las causas estructurales que dan origen al problema.

2-Entendido éste como aquel que se ocupa del desarrollo histórico de las relaciones sociales y del modo en que la historia oculta el interés y el papel activo del ser humano.
La investigación se estructura a partir de las siguientes etapas: a) momento heurístico (definición de la perspectiva teórica); b) definición del marco categorial; c) confrontación de las categorías consideradas ${ }^{3}$; d) síntesis del proceso analítico desarrollado, tomando como hilo conductor el desglosamiento de las categorías que conforman el marco categorial de la crisis ambiental en el contexto globalizado.

A partir de análisis teórico desarrollado, se intenta realizar un aporte tendiente a enriquecer los argumentos que dan cuenta del origen causal y del contexto social en que se produce el desarrollo y la profundización de la crisis ambiental, lo cual se espera constituya una contribución al campo de las ciencias socioambientales.

\section{La conformación de un marco categorial de la crisis ambiental}

Acorde con lo anteriormente señalado y a fin de avanzar en la conformación del marco categorial propuesto, se considera de especial interés la posición adoptada por diferentes autores del pensamiento social crítico, tales como: (Bauman, 2000, 2005; Beck, 1992, 1995; Caride y Meira, 2001; Guimarães, 2002; Leff, 2002; Kapp, 1995; Mayorga, 2006; Redclift, 2000 y Riechmann, 2005), los cuales, más allá de las diferencias conceptuales propias de los diversos ámbitos de los que provienen, coinciden en señalar la relación entre las características del contexto social identificado como modernidad avanzada y la profundización de la ruptura ambiental, centrando el eje de la discusión en la crisis del sistema socioeconómico dominante.

\footnotetext{
3- En cada una de las categorías analíticas propuestas, se confrontan las construcciones teóricas desarrolladas con el posicionamiento adoptado por diferentes referentes teóricos del pensamiento ambiental crítico, con el objeto de validarlas.
} 
Bajo estas condiciones, las medidas que se adoptan, con frecuencia de carácter técnico normativo, resultan insuficientes para afrontar los problemas ambientales. Por el contrario, los procesos de degradación socioambiental aceleran su ritmo a medida que se imponen y expanden las tesis del neoliberalismo, se desregulan las acciones del mercado global y el Estado pierde su capacidad de control (Agoglia, 2011).

Desde esta perspectiva, la ruptura ambiental se concibe como producto de la acción de un mercado que ignora los impactos negativos que provoca, reduciéndolos a meras externalidades de solución científico tecnológica (Kapp, citado en Agoglia, 2011). Contrariamente, el pensamiento ambiental crítico propone analizar la realidad ambiental develando las contradicciones del sistema, con el objeto que se vislumbren los componentes éticos e ideológicos que se encuentran implícitos en la crisis ecológica y se pongan de manifiesto las relaciones de poder que subyacen a la problemática socioambiental, estimulando prácticas democráticas y participativas en los asuntos públicos que intervienen en su resolución (Agoglia, 2011).

Según lo expresado, la definición del marco categorial propuesto se organiza acorde con el siguiente esquema.

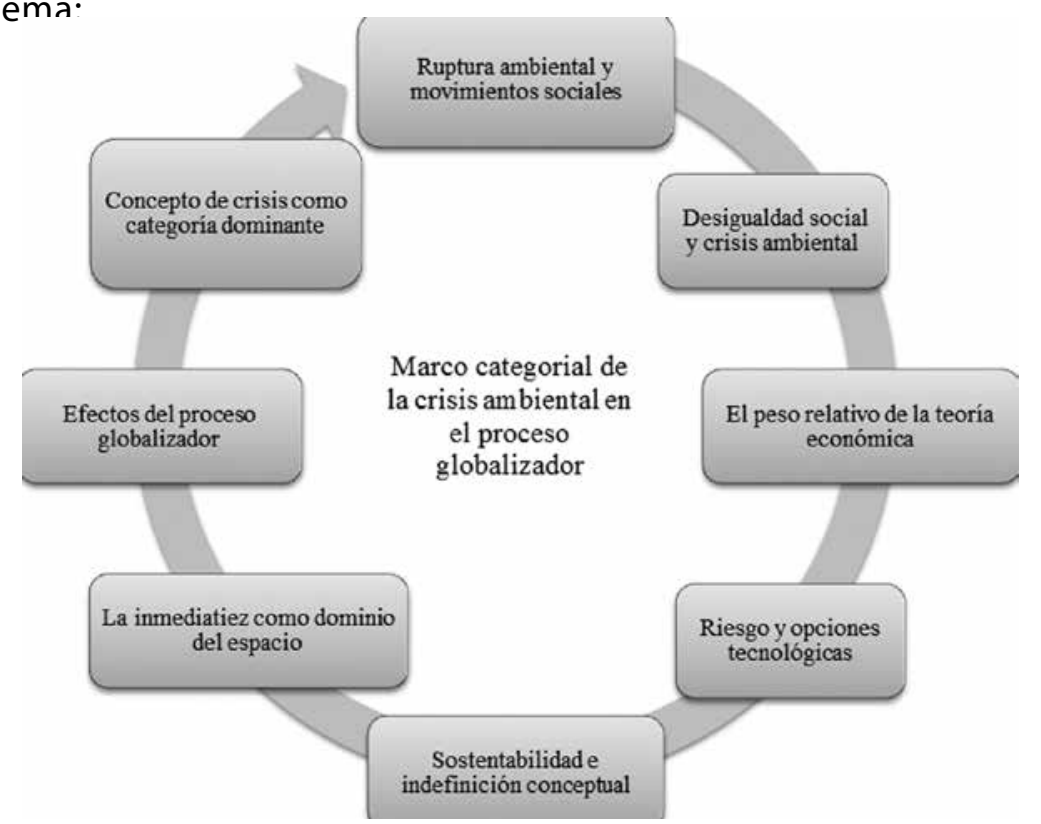

Figura 1: La ruptura ambiental y la emergencia de movimientos sociales. Fuente: Elaboración propia, sobre la base de Agoglia (2011).

El sistema neoliberal implementado desde la consolidación del modelo de consumo, promueve un proceso de fuertes desequilibrios socioambientales, que por las características que reviste se puede definir como ruptura ambiental. Kapp ${ }^{4}$ (1995), señala al respecto que:

el término ruptura ambiental engloba a todos aquellos fenómenos que afectan la calidad del medio ambiente social y natural del hombre y que tratan sobre cuestiones, que alcanzan al núcleo de la existencia humana y que en su complejidad trascienden el campo de acción y la competencia de cualquier disciplina en particular, razón por la cual, es preciso analizar las cadenas causales

4- Considerado uno de los principales economistas del Siglo XX, fuerte crítico de la compartimentalización de las ciencias sociales, fundador de las bases de la economía ecológica. 
que ocasionan dicha ruptura, teniendo en cuenta que, solamente si se observa correctamente el proceso de causación se puede esperar progresar en la tarea de controlar, o al menos limitar, sus efectos más destructivos. (p., 130).

Este proceso de desequilibrio, provoca la reacción de diversos sectores del ámbito científico y social que surgen como respuesta al uso y manejo de los recursos naturales inducido desde el modelo de crecimiento ilimitado y a las desigualdades sociales que conlleva la aplicación del binomio desarrollo-subdesarrollo.

Dicha reacción, se concentra en la emergencia de nuevos movimientos sociales identificados por la defensa de la supervivencia, la emancipación y la autodeterminación, así como por su oposición a la concentración del poder y al modelo de producción de conocimiento legitimador del sistema socioeconómico.

En este marco, la acción del movimiento ecologista se hace cada vez más notoria, ampliando su mirada desde posiciones proteccionistas y conservacionistas a una concepción más integral que engloba las distintas esferas del ámbito social.

\section{Desigualdad social y crisis ambiental}

El orden económico internacional globalizado, lejos de mitigar las desigualdades sociales y los desequilibrios ambientales profundiza la brecha centro- periferia, circunstancia que, según observa Riechmann (2005), se encuentra estrechamente relacionada con el desequilibrio que se produce entre aquellos países que viven de su ecosistema y los que explotan los recursos de toda la biosfera. Mientras los primeros, identificados como culturas ecosistémicas, manifiestan interés en proteger sus recursos debido a que su supervivencia depende de los recursos de una bioregión, una cuenca fluvial y unos pocos ecosistemas, por el contrario, las culturas biosféricas se desarrollan conjuntamente con la expansión imperialista, acopiando los recursos de su propio territorio y de los ecosistemas lejanos con la certeza de que una vez explotada una zona se puede explotar la siguiente, generando que los pueblos que viven de su ecosistema cuenten cada vez con menos recursos para ellos.

Esta consideración, pone de manifiesto que la desigualdad social y la crisis ambiental se encuentran estrechamente relacionadas y que la distribución de los bienes y los males ecológicos así como las posibilidades de mitigar sus efectos, son diferentes según los grupos sociales y la situación relativa de los países.

En este sentido, "la aceptación efectiva y consecuente de los límites del planeta y la superación de la desigualdad entre las diversas sociedades que lo habitan (y dentro de ellas), son problemas interdependientes" (Riechmann, 2005: 216).

Situación que evidencia que tanto la apropiación desigual de los bienes ecológicos: recursos naturales, flujos de energía y materiales, como la exposición desigual a los males ecológicos: enfermedades causadas por contaminación, catástrofes naturales, remiten a cuestiones de justicia ecológica. Según esta perspectiva, la mayoría de los temas de importancia ecológica están condicionados por factores económicos específicos y tienen consecuencias particulares diferentes, según la clase, 
la raza o el género. Por este motivo, es necesario tener en cuenta el papel que desempeñan las multinacionales, los grandes centros de poder global, las clases dominantes nacionales y la banca privada, que son quienes toman la mayoría de las decisiones sobre la explotación de los recursos naturales, sobre dónde se debe invertir y las estrategias económicas que se deben aplicar, de lo que resulta que, el análisis de las estructuras de poder es fundamental para entender las causas y consecuencias de la crisis ambiental (Petras, 1994 citado en Riechmann, 2005).

Desde siempre los desequilibrios en el poder y la riqueza se traducen en pautas de apropiación desigual tanto del trabajo humano como de los bienes naturales, desigualdades que, se exacerban bajo las relaciones capitalistas de producción, constituyendo la apropiación de la energía y el cálculo de la huella ecológica, dos de los ejemplos más relevantes de esta situación.

En este sentido, mientras el control de los combustibles fósiles desempeña un papel central en la ampliación de las diferencias de poder y riqueza que caracteriza la historia moderna, la huella ecológica hace referencia al área de la tierra y mar ecológicamente productiva que se requiere para proveer todos los recursos materiales, la energía consumida y para absorber todos los residuos producidos por una población determinada, con el actual nivel tecnológico, sea donde sea que se encuentre esta área (Riechmann, 2005).

A través de esta metodología se puede conocer si una sociedad consume más allá de sus límites biofísicos, es decir, si su huella ecológica excede su propio territorio, si se está apropiando de la capacidad de sustentación de otras sociedades o está abusando de bienes libres, provocando un empeoramiento de las condiciones ambientales 5 .

Situación que desde la economía clásica suele identificarse como externalización de las consecuencias negativas, en la medida que las ventajas se internalizan y las desventajas se externalizan las sociedades se estructuran en ganadoras - perdedoras, garantizando que las consecuencias positivas se desarrollen en la cima y las negativas en la base. Dicha externalización puede tener lugar en una dimensión temporal, espacial o social, es decir, desde el presente al futuro, desde el centro a la periferia o desde las clases altas a las bajas (Sachs, 1997).

\section{El peso relativo de la teoría económica}

Las condiciones señaladas, ponen en evidencia la interrelación entre problemática ambiental y modelos de desarrollo en el marco de un contexto social de crisis estructural, evidenciada como producto de una conciencia de los límites civilizatorios. Dicha conciencia se contradice con las herramientas teóricas que legitiman y dan sustento a la praxis económica dominante, cuyo peso relativo sigue imponiendo el ritmo y las condiciones del sistema social de fin de siglo.

En este sentido se observa que los hábitos de pensamiento y los marcos teóricos tienden a generalizarse y a perpetuarse mucho más allá del punto en el que llegan a ser inadecuados y de hecho irrelevantes

5-Según Riechmann (2005), en los noventa el consumo humano excede la biocapacidad en una tasa del 30 por ciento, del cual el 80 es consumido por los países ricos que tienen una huella ecológica mayor a la capacidad de carga global. Esta situación indica que el daño ambiental originado por las pautas de producción y consumo de los países poderosos casi siempre afecta en mayor medida a los más desvalidos. 
para el tratamiento de los nuevos problemas, tal como sostiene Kapp (1995):

la teoría económica clásica se ha hecho cada vez más abstracta, dejando de brindar herramientas conceptuales adecuadas para el análisis de las causas y de las complejas interrelaciones que ocasionan la ruptura ambiental y los costos sociales que genera, razón por la cual, es necesario replantearse la adecuación y relevancia de los fundamentos sobre los que se sustenta (pp. 149-150).

Fundamentalmente, por el hecho que la teoría económica tradicional continúa tratando los procesos de asignación, producción, intercambio y distribución, comosiocurriesen en una esfera económica autónoma y cerrada, con efectos poco relevantes sobre el ambiente natural $y$ social, centrando el análisis en relaciones de intercambio recíprocas y voluntarias entre unidades microeconómicas.

Teniendo en cuenta estas condiciones, Kapp (1995) plantea que la ruptura ambiental no puede explicarse como un fallo de mercado, salvo que se entienda como un fallo del sistema de mercado y de la economía convencional al momento de aceptar las interdependencias $y$ cadenas causales que la originan. Por un lado, porque la esfera económica es una abstracción, al mismo tiempo que las decisiones y comportamientos que se derivan de estos intercambios no son autónomos, sino que están determinados por las unidades económicas dominantes, las cuales al poseer un interés comercial, no pueden considerarse como el resultado de elecciones libres ${ }^{6}$, por otro, porque los efectos de la producción y la distribución sobre el ambiente no pueden considerarse

6- Kapp (1995), señala que: son las propias empresas las que deciden trasladar parte de sus costos a terceras personas o a la sociedad. Por lo cual, ningún afectado por estas decisiones, puede decir que está de acuerdo en soportar sus consecuencias de forma voluntaria. nunca como insignificantes.

Portanto, la problemática ambiental no sólo pone en tela de juicio la teoría económica, sino la eficiencia del mercado como mecanismo de dirección y coordinación de las decisiones de las distintas unidades microeconómicas, teniendo en cuenta que, ni los gastos, ni los rendimientos empresariales, ni los precios, constituyen indicadores válidos para medir los costos de la ruptura ambiental.

En este sentido, "mientras la economía continúe en esta senda metodológica no existe la esperanza de realizar un análisis adecuado de la ruptura ambiental y sus costos sociales" (Kapp, 1995: 157).

\section{Riesgo y opciones tecnológicas}

Los graves accidentes tecnológicos ocurridos como resultado de la acción humana, complementariamente a los avances registrados en materia de manipulación genética, introducen un fuerte componente ético a tener en cuenta al momento de tomar decisiones con respecto a las opciones tecnológicas, lo cual, sumado al papel que asumen los sistemas expertos en la actualidad, dan lugar a una nueva conceptualización de sociedad sustentada en la categoría de riesgo ${ }^{7}$.

Desde la concepción de Beck (1992), en este proceso, las amenazas y los peligros derivados de la tecnología crecen continuamente al punto que se produce el

7- Ulrich Beck (1992), plantea el pasaje de la modernidad industrial hacia una sociedad del riesgo, como consecuencia de la confrontación de la modernidad con las consecuencias no deseadas de sus acciones. Esta sociedad implica cambios tales como: el paso de una sociedad estamental de identidades sostenidas en la etnia, la religión y el trabajo, a una de individuación cada vez mayor; en el estatuto de la ciencia, desde una situación de monopolio del saber, hacia un escenario en que las opiniones de los expertos compiten con el saber lego y con la racionalidad social. 
paso de la sociedad industrial a la sociedad de riesgo, en la cual la lógica de producir riqueza es desplazada por la lógica de la administración de riesgos. En este sentido, los problemas más graves que afectan a la humanidad, son aquellos que resultan del propio desarrollo tecno-económico.

A diferencia de los antiguos peligros, que la modernidad intentó eliminar, los nuevos peligros producidos por la modernización resultan invisibles, no se reconocen como tales, ni pueden ser abordados por personas que no sean consideradas expertas.

Estas nuevas amenazas, necesitan de algunos elementos de la ciencia, tales como, teorías, instrumentos de medición, experimentos, etc., para hacerse visibles y para poder interpretarse como tales. Acorde con ello, la sociedad del riesgo sigue siendo un modo legítimo de modernidad, en el sentido que sigue basándose en la creencia de que aplicando la razón se pueden doblegar las realidades conforme a la voluntad humana.

En este marco y dada la institucionalización del riesgo, el valor comercial potencial del temor al riesgo se vuelve infinito, según Bauman (2005), combatir riesgos se convierte en un negocio enorme y sumamente lucrativo con capacidad para perpetuarse a sí mismo ${ }^{8}$. Por su parte, tal como advierte Riechmann (2005), esta situación es producto del desfase creciente entre los avances tecno-científicos y la evolución de la sociedad.

A partir de la ruptura tecnológica de los años sesenta, el desarrollo explosivo de algunas ramas de la ciencia como la biología molecular y la informática,

8- Según Bauman (2005), aprovechando el temor al riesgo es posible conseguir lo que sea, por ejemplo en el campo de la salud, donde los riesgos generan millones de dólares. Por lo que su desaparición, provocaría un desastre comercial. provoca un desequilibrio en la estabilidad del sistema científico-técnico tornando cada vez más difícil su control por parte del poder público.

Ante esta situación, se vuelve fundamental aplicar el principio de precaución, formulado en la Conferencia de Naciones Unidas sobre el Medio Humano de 1972, sobre la base de la responsabilidad, el respeto, la prevención, la obligación de informar y en la necesidad de actuar anticipándose a los problemas, incluso en ausencia de una prueba concluyente del daño, sobre todo si existe incertidumbre científica al respecto (Riechmann, 2005).

Desde esta perspectiva, cada vez que una nueva actividad científico-técnica amenaza a la salud o al ambiente, deben tomarse medidas preventivas. Cumpliendo este principio, es obligación informar a la población sobre los posibles daños y sobre la gama de acciones posibles incluyendo la posibilidad de su no aplicación.

En este sentido, según advierte Riechmann (2005), la solución no consiste en bloquear el desarrollo de nuevos conocimientos sino en tener un control sobre ellos, evaluando las alternativas tecnológicas, dejando de lado aquellas que conllevan riesgos demasiado altos.

\section{Sostenibilidad e indefinición conceptual}

Hacia el interior del debate descrito, se plantean diversas interpretaciones sobre lo que se entiende por desarrollo sostenible, poniendo de manifiesto una crisis de identidad conceptual enmarcada en la lucha por la definición y apropiación del concepto de sostenibilidad, utilizado tanto para identificar y promover alternativas a la crisis existente, como 
para justificar la tesis neoliberal de un crecimiento económico ecológicamente tolerable.

En este marco, según observa Redclift ${ }^{9}$ (2000) , el desarrollo sostenible es un concepto cargado de contradicciones que remite a una cuestión confusa, cuya aceptación deriva precisamente de la gran cantidad de acepciones que puede tener y la forma en que se lo puede utilizar en apoyo a una gran variedad de agendas políticas y sociales.

La idea de sostenibilidad aunque deriva de la ciencia, evidencia las limitaciones de la propia ciencia, utilizándose para defender imperativos morales y humanos legitimados a partir de imperativos biofísicos, con lo cual se acentúa la idea de que su fuerza reside en su ambigüedad (Redclift, 2000).

En su análisis, Redclift (2000) hace referencia al legado histórico a partir del cual se ha fortalecido la idea de desarrollo sostenible, llegando a la conclusión de que la idea de sostenibilidad:

emerge como resultado de un conjunto de hechos específicos, comenzando con la idea de progreso, asociados con el proyecto de la ilustración, situación que arrastra serias implicancias para las estrategias globales de desarrollo y asegura la continuidad de la hegemonía económica de los países industrializados del norte (p.19).

Desde su perspectiva, a partir del siglo XVIII comienza una nueva relación del hombre con la naturaleza, influenciada por la teoría de la evolución, la especialización de las ciencias y el desarrollo económico, que definen el contexto en que la sostenibilidad

9- Michael Redclift, fue el primer director del programa de Cambio Ambiental Global entre 1990 y 1995 . En el 2006 fue galardonado con el Premio Buttel, por su contribución a la sociología ambiental. Sus temas de investigación incluyen: desarrollo sostenible, cambio ambiental global, seguridad ambiental y sistema alimentario. se convierte en un concepto importante. En este marco, existen dos elementos que definen la ambigüedad desde la que se invoca el desarrollo sostenible, por un lado, la idea de progreso, razón y libertad, por otro, la forma en que la modernidad legitima sus prácticas discursivas.

En esta misma línea de pensamiento, Guimarães (2002: 71), sostiene que "la fuerza que ha cobrado el discurso de la sustentabilidad encierra múltiples paradojas", entre ellas, la impresionante unanimidad en su favor, lo cual resulta contradictorio si se tiene en cuenta el enorme vacío que suele acompañar a los consensos sociales absolutos, más aún, considerando que la misma idea de desarrollo, emerge de la pugna de intereses contradictorios.

Las circunstancias descritas lo llevan a afirmar que más allá de una posible coincidencia en cuanto al agotamiento del sistema vigente, en la realidad, sólo se recurre a la sustentabilidad para introducir restricciones al proceso de acumulación, sin afrontar los profundos cambios político institucionales que implica la reglamentación de la propiedad, el control, acceso y uso de los recursos naturales y los servicios ambientales, del mismo modo que no se promueven modificaciones profundas en los patrones de consumo, dejando entrever que detrás de tanta unanimidad, existen intereses de poder basados en visiones particulares de lo que se entiende por sustentabilidad. Desde la perspectiva de Guimarães (2002), esta situación:

responde a una contradicción sistémica a la cual se puede denominar como conservadurismo dinámico, que sintetiza la tendencia inercial del sistema social para resistir al cambio, promoviendo la aceptación del discurso transformador 
precisamente para garantizar que nada cambie, en una suerte de gatopardismo posmoderno" (p.73).

Lo cual constituye un ejemplo de la capacidad del sistema de subsumir las contradicciones, cooptando la esencia crítica del concepto de sostenibilidad para convertirlo en algo cargado de simbolismo y vacío de contenido, con el agravante que, en la medida que desde los grandes centros de poder mundial se promueve el desarrollo sostenible, el discurso crítico pierde claridad respecto a su objeto de crítica.

\section{La inmediatez como dominio del espacio}

Según la perspectiva de Bauman:

El advenimiento y desarrollo de la modernidad pueden evaluarse empleando diferentes parámetros, sin embargo, existe un rasgo de la vida moderna que sobresale sobre el resto, como "diferencia que hace la diferencia" y como atributo del cual derivan todas sus demás características, que es el cambio en la relación entre espacio y tiempo (2000: 14).

Desde la concepción de Bauman (2000), la modernidad comienza cuando el espacio y el tiempo se interpretan como categorías independientes, dejando de considerarse como aspectos entrelazados a través de una relación de correspondencia estable.

Bajo las condiciones de la modernidad sólida, el tiempo, constituye el arma para la conquista del espacio, el cual no puede ser ampliado ni reducido, en este sentido, la velocidad como forma de dominio se convierte en uno de los objetivos principales de la ciencia y la técnica moderna.
El espacio constituye el aspecto pesado e inerte mientras que el tiempo simboliza el aspecto ágil y dinámico. Por el contrario en la etapa líquida, la aceleración del tiempo alcanza la máxima velocidad posible llegando a su límite natural. Al reducir el tiempo a la instantaneidad y al lograr desplazarse con la velocidad de la señal electrónica, el poder se vuelve extraterritorial, en la medida que ya no se encuentra atado a la resistencia del espacio (Bauman, 2000).

Esta característica otorga una ventaja para quienes detentan el poder, consistente en la capacidad de ubicarse fuera del alcance de las personas sobre las que ejercen su dominio volviéndose inaccesibles.

Según esta perspectiva, las nuevas técnicas de poder se encuentran en la capacidad de huida, el escurrimiento, el rechazo a lo concreto y a la responsabilidad, sin tener la necesidad de afrontar los costos ni las consecuencias de sus acciones. En este contexto el objetivo no es la conquista del territorio, sino la ruptura de las trabas que condicionan el accionar del poder globalizado, "la guerra de hoy se parece cada vez más a la promoción del libre comercio mundial por otros medios" (Bauman 2000: 17).

Bajos estas circunstancias, la élite global sigue el esquema de los antiguos amos ausentes, gobernar sin cargarse de las responsabilidades administrativas, evitando establecer cualquier tipo de relación estable con las poblaciones subordinadas, desde una perspectiva desde la cual, el compromiso se convierten en algo costoso y poco efectivo.

En el universo líquido el espacio puede recorrerse en una fracción de tiempo, dejando de constituir una traba y un límite 
para la acción del poder global y perdiendo su valor estratégico, en el sentido que si todos los espacios pueden alcanzarse al mismo tiempo, ningún espacio tiene un valor especial.

La dominación se identifica con las fuentes de incertidumbre, representadas por aquellos que consiguen mantener sus actos libres e impredecibles. La nueva dominación consiste en la capacidad de escapar y descomprometerse, despojando a los dominados de su capacidad de imponer límites a su acción. Bajo estas condiciones, los individuos que actúan y se mueven más rápido, los que más se acercan a la instantaneidad y el movimiento, son los que ejercen el poder.

Según sostiene Bauman, "el capital se convierte en algo volátil e inconstante, su extraterritorialidad le permite desplazarse libremente, constituyendo su liviandad la mayor fuente de incertidumbre $y$ dominación y el factor principal de división social" (2000: 130).

La nueva fuente de ganancias consiste en la desenfrenada velocidad de circulación, de reciclado, envejecimiento, descarte y remplazo, en contraposición a la durabilidad y a la confiabilidad del producto.

En un contexto social en que la infinidad de posibilidades ha despojado al tiempo de su poder de seducción, la durabilidad pierde su atractivo, pasando de ser un logro a una desventaja. El corto plazo remplaza al largo plazo convirtiendo a la instantaneidad en ideal último.

\section{Los efectos del proceso globalizador}

El fin del siglo XX plantea un mundo que parece haberse quedado sin opciones, la multiplicidad de dimensiones que conforman el proceso globalizador rebasan los marcos referenciales sobre los que se sustenta la racionalidad dominante, dando como resultado una sensación de incertidumbre y ambivalencia producto de la profundización de los valores modernos.

Esta situación, provoca la emergencia de movimientos sociales enmarcados en la lucha contra la homogeneización socioeconómica y el pensamiento único, generando un profundo debate teórico hacia el interior de las ciencias sociales y humanas.

Siguiendo esta línea argumentativa, Riechmann (2005), sostiene que la fractura de las dimensiones espacio-tiempo, resultado de los avances tecnológicos, sumado a la extraterritorialidad del poder, lleva a un incremento de la desigualdad como resultado de la independencia creciente de las elites globales de los poderes políticos y culturales territorialmente acotados, provocando una redistribución del poder en beneficio de las elites globales y una asimetría entre la naturaleza extraterritorial del poder y el carácter territorial de la vida en su conjunto.

Desde su posición, los precios bajos de los recursos naturales con relación al de los productos manufacturados permiten a los países industrializados apropiarse de grandes cantidades de recursos biofísicos manteniendo al mismo tiempo relaciones de comercio exterior equilibradas en términos monetarios. Es decir que, lo que dentro del sistema de precios se concibe como un comercio de tipo equitativo, es en realidad un intercambio desigual en términos biofísicos que obliga a los más débiles a explotar, sobre explotar o incluso agotar sus recursos, mientras los 
países ricos mantienen una mayor calidad ambiental.

En este sentido, la globalización es un término ambiguo que encierra dos ideas antagónicas, por un lado la globalización empresarial que permite que las empresas compitan sin trabas en los mercados mundiales, por otro, la globalización democrática y la conformación de instituciones globales que garanticen la pluralidad de culturas y reconozca los derechos fundamentales de cada ciudadano del mundo.

Bajo las condiciones antedichas, el poder económico se desvincula de sus obligaciones sociales, con las generaciones futuras y en general con las condiciones de reproducción de la sociedad, siendo esta ausencia de responsabilidades una de las principales estrategias para mantener su condición (Riechmann, 2005).

Las dimensiones descritas se encuentran estrechamente relacionadas con la profundización de la ruptura ambiental y con la evidente contradicción que surge entre sus crecientes manifestaciones y las estrategias de solución propuestas desde la Declaración de la Tercera Cumbre de la Tierra, desarrollada en el año 2002, cuyos resultados ponen de manifiesto las relaciones de poder que atraviesan el discurso ambiental dominante, desviando la atención de las responsabilidades que le caben a cada uno de los países hacia posicionamientos desde los cuales la mayor responsabilidad recae sobre los individuos, lo cual implica un retroceso en los avances obtenidos a nivel del debate conceptual, como en los logros alcanzados en materia de participación social y horizontalidad en la toma de decisiones.

Según la concepción de Bauman (2000), esta situación responde a la intromisión de la esfera privada en el espacio público, uno de cuyos efectos es la desintegración de los vínculos sociales y del concepto de ciudadanía, lo que sumado a la ausencia de valores sólidos de los cuales aferrarse, conlleva a que las responsabilidades sociales recaigan sobre los hombros de los individuos, reforzando la sensación de incertidumbre que genera el proceso de individuación en la modernidad avanzada.

En este sentido, la desintegración de la trama social y el desmoronamiento de las fuentes de acción colectiva, se señalan como producto de un poder que cada vez resulta más cambiante y evasivo, acorde con sus actuales características de falta de compromiso y capacidad de huida.

\section{El concepto de crisis como categoría dominante}

El acrecentamiento de la pobreza y la agonía del Planeta, sumado al estilo de vida uniformador y hedonista propuesto por la sociedad de consumo, ponen en cuestionamiento las bases sobre las que se sustenta el modelo económico dominante, dando lugar a una situación de crisis que marca el inicio del proceso de ruptura del proyecto moderno ${ }^{10}$.

El crecimiento indefinido basado en la racionalidad del mercado y en las capacidades de innovación tecnológica, se enfrenta a una situación paradojal que refleja los alcances de una crisis cargada de evidencias sobre los efectos de la razón instrumental sobre el sistema natural y de incertidumbre sobre las soluciones a emprender.

10- Dussel (2000), lo define como el último estadio de la historia de la globalización, argumentando que esta ha pasado por cuatro estadios, de los cuales el último es el que sitúa a Europa y a la cultura occidental como centro. 
La razón como base de la irracionalidad y la ambivalencia como fruto del progreso, constituyen las contradicciones existenciales de las sociedades contemporáneas poniendo de manifiesto el estado de crisis en que se encuentran, ante las incertidumbres que conlleva la ruptura del proyecto moderno.

Bajo esta perspectiva la crisis contemporánea, según Touraine (1993), puede ser identificada como una crisis de inteligibilidad, lo cual hace evidente la distancia entre lo que sería esencial comprender y las herramientas intelectuales necesarias para tal comprensión. En este sentido, según la posición de Leff:

La crisis ambiental es la crisis de nuestro tiempo... pero, también, crisis del pensamiento occidental, de "determinación metafísica" que, ... abrió el camino para la racionalidad científica e instrumental que produjo la modernidad como un orden cosificado y fragmentado, como formas de dominio y control sobre el mundo. Por eso, la crisis ambiental es sobre todo un problema de conocimiento" (2002: 191).

Para intentar explicar este proceso, diversos analistas de la sociedad occidental interpretan la crisis contemporánea y su problemática emergente, como un síntoma de la declinación de un modelo social que no encuentra salidas para resolver sus contradicciones en el marco del actual sistema normativo. Ejemplo de ello se manifiesta en la obra de Sábato cuando señala,

...esta crisis no es la crisis del sistema capitalista como muchos imaginan, es la crisis de toda una concepción del mundo y de la vida basada en la idolatría de la técnica y en la explotación del hombre... donde tener poder significó apropiarse y la explotación llegó a todas las regiones posibles del mundo (2000: 83).
Situación que pone en evidencia la necesidad, de analizar las contradicciones de un proyecto moderno, que tal como expresa Mayor Zaragoza (2000), incluye tanto los avances del progreso y la civilización como la posibilidad de su destrucción irreversible.

\section{Síntesis del proceso analítico desarrollado}

Del análisis desarrollado se concluye que el sistema neoliberal implementado por el modelo de consumo, promueve un proceso de fuertes desequilibrios socioambientales, que por las características que reviste se puede definir como una etapa de crisis estructural.

En el curso del proceso de modernización, las amenazas y los peligros de los poderes de la tecnología crecen continuamente al punto que se produce el paso de la sociedad industrial a la sociedad del riesgo. En este sentido, los problemas más graves que afectan a la humanidad y a los que debe hacer frente la tecnología, son justamente los que resultan del propio desarrollo tecno-económico.

El fin del siglo XX plantea un mundo que parece haberse quedado sin opciones, la multiplicidad de dimensiones que conforman el proceso globalizador rebasan los marcos referenciales sobre los que se sustenta la racionalidad dominante, dando como resultado una sensación de incertidumbre y ambivalencia producto de la profundización de los valores modernos.

Se produce una crisis de identidad conceptual, que responde a una contradicción sistémica que sintetiza la tendencia inercial del sistema social para resistir al cambio, promoviendo la 
aceptación del discurso transformador precisamente para garantizar que nada cambie.

El orden económico internacional globalizado, lejos de mitigar las desigualdades sociales y los desequilibrios ambientales profundiza la brecha centroperiferia, circunstancia que se encuentra estrechamente relacionada con el desequilibrio existente entre aquellos países que viven de sus ecosistemas y los que explotan los recursos de toda la biosfera.

La desigualdad social y la crisis ambiental se hallan estrechamente relacionadas, constituyendo la apropiación de la energía y el cálculo de la huella ecológica, sus ejemplos más elocuentes.

La inmovilidad social responde a una contradicción sistémica a la cual se puede denominar como conservadurismo dinámico, esta situación constituye un ejemplo de la capacidad del sistema de subsumir las contradicciones, cooptando la esencia del discurso crítico para convertirlo en algo cargado de simbolismo y vacío de contenido.

La fractura de las dimensiones espacio-tiempo, resultado de los avances tecnológicos, sumado a la extraterritorialidad del poder, lleva a un incremento de la desigualdad como resultado de la independencia creciente de las elites globales de los poderes políticos y culturales territorialmente acotados, provocando una redistribución del poder en beneficio de las multinacionales y una asimetría entre la naturaleza extraterritorial del poder y el carácter territorial de la vida en su conjunto.

La contradicción que surge entre las manifestaciones de la crisis ambiental y las estrategias de solución propuestas, pone de manifiesto las relaciones de poder que atraviesan el discurso ambiental dominante, desviando la atención de las responsabilidades globales hacia posicionamientos desde los cuales la mayor responsabilidad recae sobre los individuos, lo cual implica un retroceso en los logros alcanzados en materia de participación social y horizontalidad en la toma de decisiones. En este sentido, la actual problemática ambiental no sólo pone en tela de juicio la teoría económica, sino las decisiones adoptadas desde las distintas unidades microeconómicas, haciendo evidente que ni los gastos, ni los rendimientos empresariales, ni los precios, constituyen indicadores válidos para medir los costos de la ruptura ambiental.

Acorde con sus características y nivel de profundización, la crisis ambiental plantea una disyuntiva en torno a las alternativas de solución propuestas. Entre aquellas posiciones que consideran que se pueden ofrecer soluciones viables sin necesidad que se produzcan cambios en las estructuras del sistema de mercado, fundamentalmente a partir del establecimiento de políticas basadas en el desarrollo tecnológico, la iniciativa privada y el control normativo, y las que por el contrario, plantean soluciones sustentadas en la implementación de modelos alternativos de producción y consumo, enfocando el eje en la necesidad de replantear el valor económico asignado a los bienes naturales, así como en la adopción de opciones tecnológicas de libre transferencia.

La multiplicidad de dimensiones que se desprenden de este proceso, rebasan los marcos referenciales de la racionalidad dominante, generando un profundo debate teórico, desde el cual, la dominación 
del orden económico, la ruptura de las relaciones espacio-temporales, la disolución de los vínculos sociales y la extraterritorialidad del poder, se señalan como las características más relevantes y cuya principal capacidad se sustenta en convertir al resto de los ámbitos sociales en instrumentos del accionar económico, con el objeto de mantener el imperativo de expansión y el nivel creciente de acumulación de ganancias.

Bajo esta concepción, las leyes naturales que rigen el universo físico se equiparan a las leyes naturales del mercado, las cuales movidas por la libre iniciativa y el egoísmo individual, rigen el funcionamiento del universo económico. Como consecuencia de ello, cualquier tipo de acción se torna válida sea moral o no, al quedar justificada por su resultado.

El crecimiento económico se institucionaliza como base del desarrollo, desde una perspectiva que reduce el concepto de desarrollo a las magnitudes económicas, convirtiéndose en la razón de ser de todos los desarrollos posibles, incluido el personal y el social.

En este proceso, la razón se transmuta en razón instrumental, convirtiéndose en un mecanismo legitimador y un instrumento más al servicio del mantenimiento de las relaciones desiguales de poder.

Acorde con todo lo expuesto, la alternativa propuesta se estructura en torno a la reconfiguración del espacio público como lugar de encuentro y debate, reconectando las necesidades individuales con los intereses colectivos, recuperando el rol de la ciudadanía, a partir del accionar del pensamiento crítico, cuyo principal objeto es hacer visibles aquellos dispositivos que dificultan la conformación de una sociedad en la que los individuos se perciban como parte de un objetivo común.

Si se considera que siempre hay causas que explican lo que se nos muestra como realidad, el objetivo es desentrañar esas causas y cuestionar los mecanismos de reproducción en que se apoya el sistema, teniendo en cuenta que al conocer el funcionamiento de estos mecanismos existen mayores posibilidades de minimizar su acción reproductora. De lo cual se concluye que la comprensión crítica de la crisis ambiental constituye un punto de partida inexcusable para las actuales teorías del conocimiento, no sólo para interpretarla en toda su extensión, sino para desarrollar estrategias alternativas de resolución. 


\section{REFERENCIAS}

Agoglia, O. (2012). La incidencia del proceso de instrumentalización de la razón sobre la ruptura del proyecto moderno y la emergencia de la crisis ambiental.. En Arias, M.A \& Camacho, C. (eds), Educación y formación ambiental: algunos escenarios en la educación superior (pp. 105-126). Sinaloa, Universidad Autónoma de Sinaloa - Universidad Autónoma de la ciudad de México, Consejo Estatal de Ciencia y Tecnología e Instituto de Investigación y Defensa del Ambiente.

----- (2011). La crisis ambiental como proceso. Un análisis reflexivo sobre su emergencia, desarrollo y profundización desde la perspectiva de la teoría crítica. Tesis de Doctorado, Girona: Universitat de Girona.

Bauman, Z. (2000). Modernidad Líquida. Buenos Aires: Fondo de Cultura Económica.

----- (2005). Ética posmoderna. Buenos Aires: Siglo XXI editores.

Beck, U. (1992). La sociedad del riesgo. Madrid: Siglo XXI editores.

----- (1995). Políticas ecológicas en la edad del riesgo. Barcelona: El Roure.

Caride, J. \& Meira, P. (2001). Educación ambiental y desarrollo humano. Barcelona: Ariel.

Commoner, B. (1973). El círculo que se cierra. Barcelona: Plaza y Janés.

Dussel, E. (2000). Para una ética de la liberación latinoamericana. Buenos Aires: Siglo XXI editores.

Guimarães, R. (2002). La ética de la sustentabilidad y la formulación de políticas de desarrollo. En Alimonda, H. (Ed). Ecología Políticas, Naturaleza, Sociedad y Utopía. Buenos Aires: CLACSO.

Horkheimer, M. (1947). Crítica a la razón instrumental. Buenos Aires: Ser.

Kapp, K. (1995). La ruptura ambiental, un desafío para las Ciencias Sociales. En. Aguilera F. Economía de los recursos naturales: un enfoque institucional, Madrid: Visor-Fundación Argentaria.

Leff, E. (2002). Saber Ambiental. Sustentabilidad, racionalidad, complejidad, poder. Buenos Aires: Siglo XXI editores.

Mayor, F. (2000). Un nuevo mundo. Barcelona: Galaxia Gutenberg.

Mayorga, E. (2006). Teoría crítica y crítica política en la cuestión ambiental: problemas y perspectivas. En Alimonda, H. (Ed). Los tormentos de la materia. Aportes para una ecología política latinoamericana. Buenos Aires: CLACSO. 
Redclift, M. (2000). El desarrollo sostenible, necesidades, valores, derechos. En: Bárcena, I.; Ibarra, P. \& Subyaga, M. (Eds.). Desarrollo sostenible un concepto polémico. Bilbao: Universidad del País Vasco.

Reyes Mate, M. (1998). Las Escuelas de Fráncfort o un mensaje en una botella. Boletín Informativo de la Fundación Juan March, № 285: 497-512.

Riechmann, J. (2005). Un mundo vulnerable. Ensayos sobre ecología, ética y tecnociencia. Madrid: Los libros de la Catarata.

Sábato, E. (2000). La Resistencia, Barcelona: Seix Barral.

Sachs, W. (1997). Diccionario del desarrollo: una guía del conocimiento como poder. Cochabamba: PRATEC-CAI.

Touraine, A. (1993). Crítica de la modernidad. Madrid: Temas de hoy. 